\title{
REVIEW
}

\section{Inherited susceptibility for aggressive prostate cancer}

\author{
William B Isaacs
}

\begin{abstract}
Whether or not there is inherited basis for prostate cancer aggressiveness is not clear, but advances in DNA analysis should provide an answer to this question in the very near future.
\end{abstract}

Asian Journal of Andrology (2012) 14, 415-418; doi:10.1038/aja.2011.146; published online 30 April 2012

Keywords: aggressiveness; germline; Genome Wide Association Studies; GWAS; inherited; linkage; mutation; single nucleotide polymorphisms; SNPs; susceptibility

\begin{abstract}
INTRODUCTION
The development of prostate cancer, similar to other common diseases, is influenced by both genetic and environmental factors. Migration studies documenting increased incidence of prostate cancer in Asian men moving to the United States is a clear example of environmental effects on prostate cancer risk. On the other hand, the demonstration that prostate cancer tends to cluster in families, and increased concordance rates for prostate cancer among monozygotic $v s$. dizygotic twin pairs are consistent with an important genetic determinant for prostate cancer risk. ${ }^{1,2}$ Indeed, tremendous progress has been made over the past several years identifying genetic risk factors for this common cancer. A fundamental question, particularly from a clinical perspective still remains: are there inherited determinants of prostate cancer progression and/or aggressiveness as well? This chapter will provide a brief overview of studies aimed at defining inherited mechanisms for prostate cancer risk, and in particular, efforts to understand inherited risk for more aggressive prostate cancer.
\end{abstract}

\section{GENETIC BASIS FOR PROSTATE CANCER}

Inherited susceptibility for a common cancer like prostate cancer reflects a diverse spectrum of genetic influences. At one end of this spectrum are genes with rare, high penetrance variants or alleles that have a strong influence on the development of disease. Examples are the BRCA1 and $B R C A 2$ genes which can be associated with very large increases in breast cancer risk compared to the general population. ${ }^{3}$ For prostate cancer, many family-based linkage studies have sought evidence for high penetrance genes, albeit with only limited success. ${ }^{4}$ While these studies have not ruled out a role for this class of genes in prostate cancer susceptibility, they do indicate that only a small proportion of prostate cancer cases is likely the result of the action of such genes. The recent finding ${ }^{5}$ of rare mutations in the prostate-specific transcription factor, HOXB13, which are associated with large increases in risk of early-onset, familial prostate cancer has provided renewed excitement for the identification of more rare, moderate-to-high penetrance prostate cancer susceptibility alleles, particularly given the emerging opportunities for disease gene identification made possible through next-generation DNA sequencing. Indeed, it will be of considerable interest to see to what extent this class of genes can account for familial clustering of prostate cancer, and for prostate cancer susceptibility in general.

At the other end of the genetic spectrum are more common (allele frequencies: about 5\%-50\%), low penetrance alleles that have a more modest association with the disease phenotype, being associated with only fractional increases in risk. The 'Common Disease Common Variant' hypothesis ${ }^{6}$ that proposed that common diseases like prostate cancer might have common genetic determinants was a strong stimulus for the HapMap project, and the characterization of tagging singlenucleotide polymorphisms (SNPs) that could be used as surrogates for complete genome sequencing to capture the majority of genetic variation in a given population. Panels of tagging SNPs were used to develop high through-put genotyping platforms which in turn paved the way for Genome Wide Association Studies (GWAS), where allele frequencies at hundreds of thousands of SNP loci (recent chip designs feature millions of SNPs) across the genome are compared, typically using a case-control design. By systematically and objectively searching the entire genome, GWAS can identify novel disease risk alleles in the genome. Among the over 1400 trait loci recently identified for a wide variety of human diseases and phenotypes using GWAS, ${ }^{7}$ prostate cancer is well represented in having more than 40 reproducible loci found since 2008 , primarily by groups in Iceland, England and the United States. ${ }^{8-14}$ While these studies have provided support for genetic variation in the regulation of a number of genes already implicated in prostate cancer biology, including MYC, NKX3.1, MSMB and PSA, a number of novel genes and thus novel pathways have been implicated by these studies as well, including HNF1B, TET2 and NUDT10/11.

Although individually the variants around these and other genes have very modest effects on prostate cancer risk with increases of about $15 \%$ $30 \%$, their cumulative effect can be much more substantial. Zheng et al. ${ }^{15}$ demonstrated that three SNPs on chromosome 8q and two variants on chromosome 17 had a cumulative effect on prostate cancer risk. Men who carried four or more risk alleles at these SNP loci had substantially increased risk of prostate cancer ( $\mathrm{OR}>4$, as compared with non-carriers). More recently, Kote-Jarai et al. estimate that men in the top $1 \%$ of a genetic risk distribution have approximately three to four times the risk of developing prostate cancer compared to the average population. ${ }^{16}$

The Brady Urological Institute, Johns Hopkins Medical Institutions, Baltimore, MD 21287, USA

Correspondence: DrWB Isaacs (wisaacs@jhmi.edu)

Received: 16 March 2012; Revised: 30 March 2012; Accepted: 30 March 2012; Published online: 30 April 2012 
Unfortunately, however, these prostate cancer risk-associated SNPs have only limited ability to distinguish risk for more or less aggressive cancer, ${ }^{17}$ consistent with the idea that most of the susceptibility loci identified to date impact aspects of disease initiation rather than disease progression. $^{16,17}$

\section{AGGRESSIVE VS. NON-AGGRESSIVE PROSTATE CANCER}

It is well appreciated that prostate cancer is the most commonly diagnosed cancer in men in the United States and a number of other countries. A fact becoming more widely appreciated is that most aged men in the United States have prostate cancer-that is, they have cells in their prostates that if observed on a needle biopsy would be diagnosed as prostate cancer. Support for this conclusion comes from the autopsy studies of Powell et al. that showed that prostate cancer could be found in $50 \%$ of 50 -year-old men, and $70 \%-80 \%$ of men over age 70 years, dying from non-prostate cancer-related causes. ${ }^{18}$ In the past, these lesions were called 'microscopic' or 'latent' foci of prostate cancer, indicating that they were typically small, non-progressive, clinically insignificant and rarely detected through routine prostate cancer screening. However, this latter notion has been strongly challenged by numerous studies, with the Prostate Cancer Prevention Trial finasteride study being an informative example. ${ }^{19}$ This study enrolled men who were considered at relatively low risk for prostate cancer by virtue of the study entry criteria of having a serum prostate-specific antigen (PSA) values less than $3 \mathrm{ng} \mathrm{ml}^{-1}$, and who were over the age of 55 years. A critical and unique aspect of this study was that all men were biopsied at the end of the trial regardless of PSA or digital-rectal examination status. In the control (placebo) arm of the study, $\sim 25 \%$ of the over 4000 biopsied men (mean age: 63 years) were diagnosed with prostate cancer. This number of cases was four times higher than originally anticipated, and indicates that as many as one in four 60 -year-old men in this country would be diagnosed with prostate cancer if biopsied. This figure could be even higher as most of the men biopsied in the Prostate Cancer Prevention Trial trial were subjected to sextant biopsies, whereas the routine use of 12 or 18 biopsies to sample larger amounts of prostate tissue is becoming widespread. It is not beyond reason to assume that with the development and implementation of more aggressive and effective prostate screening strategies that the clinical diagnosis rate could begin to approach the theoretical incidence rates of prostate cancers found at autopsy described above. Thus, with widespread PSA screening, only a small percentage of prostate cancer diagnoses are life threatening. Some of these patients do not even need to be treated.

In contrast to this much publicized overtreatment problem, every $20 \mathrm{~min}$ a man in the United States dies from prostate cancer, resulting in over 28000 deaths annually. ${ }^{20}$ This subset of patients with an aggressive form of prostate cancer can only be successfully treated by aggressive, early treatment. While it is important to have effective means to diagnose men with prostate cancer, it is probably at least as important to identify men at increased risk of aggressive disease, for early detection and definitive treatment. Genetic markers which can be assayed non-invasively and inexpensively and are not chronologically dependent upon the disease process itself to be informative may provide an ideal means of addressing this need.

\section{GENETIC PREDISPOSITION TO AGGRESSIVE PROSTATE CANCER}

As discussed above, recent breakthroughs through GWAS have led to the discovery of a panel of SNPs that are associated with prostate cancer risk by comparing men with or without prostate cancer using case-control designs. ${ }^{8-14}$ These findings have stimulated much discussion about the clinical utility of such SNPs, particularly in combination, as a risk stratification tool. ${ }^{21-23}$ However, as mentioned above, these prostate cancer risk-associated SNPs have only limited ability to distinguish risk for more or less aggressive cancer; ${ }^{16,17}$ thus, enthusiasm for translation of these markers to the clinic has been limited. ${ }^{24}$ An important caveat is that many GWAS performed to date have compared SNP allele frequencies in all prostate cancer cases to those in controls, and most cases, particularly in populations subjected to extensive PSA-based disease screening, will be clinically localized disease with low likelihood for progression. To increase power to detect genetic determinants of aggressive disease, it may be necessary to carry out comparisons of large numbers of cases with more aggressive disease to cases with less aggressive disease, as opposed to comparing all cases to controls.

Is there a basis to expect that a genetic predilection to develop more aggressive prostate cancer exists? Relatedly, is there any epidemiological evidence to support an inherited influence on prostate cancer aggressiveness? Or is genetic susceptibility restricted to the cancer initiation process, with disease progression resulting from environmental or stochastic processes driving clonal selection of somatically occurring genomic alterations? While somatic alterations undoubtedly play an important role in shaping a cancer's aggressiveness phenotype, several epidemiological studies provide support for germline determinants of tumor aggressiveness. Lindström et al. ${ }^{25}$ examined familial concordance rates for cancer survival in a Swedish population-based study. Overall, they found a concordance for prognosis for parents and children with various cancers, including prostate, consistent with a hereditary component affecting disease outcome. They noted an increased hazard ratio $(2.07$ (95\% CI: 1.13-3.79)) for death from prostate cancer in men with poor paternal survival compared with those with good paternal survival, suggesting that prostate cancer-specific survival in fathers predicts survival from the same cancer in their sons. In a more recent, related study, Hemminki ${ }^{26}$ also showed evidence for the familial concordance of good and poor survival in prostate cancer, and that familial risks were somewhat higher for fatal than for incident prostate cancer, suggesting that fatal prostate cancers may be a genetic subgroup. Importantly, this author points out that considering the high familial risks for fatal prostate cancer, family history remains an important prognostic indicator useful for clinical genetic counseling.

\section{GENETIC STUDIES FOCUSING ON AGGRESSIVE PROSTATE CANCER}

Genetic linkage studies are widely used to identify chromosomal regions and genes predisposing to a disease in families with multiple affected members. Identification of statistically significant linkage provides strong evidence for genetic susceptibility to a disease. As mentioned earlier, linkage studies of prostate cancer have been ongoing for many years, but with only limited success. ${ }^{27}$ More recent efforts in linkage studies of prostate cancer have largely shifted to address more aggressive prostate cancer as it is hoped that that this approach might reduce the locus and disease heterogeneity problems that have confounded linkage analyses, as well as focus resources on finding genes that are the most clinically relevant. ${ }^{27}$

In the past decade, more than 10 different genome-wide linkage studies of aggressive prostate cancer have been published. Initial efforts focused on the phenotype of Gleason Score ${ }^{28-34}$ as an aggressiveness indicator, implicating genomic intervals at 5q31-33, 7q32, and $19 q 12-13.11$ as harboring aggressive prostate cancer loci. Using a definition of aggressive prostate cancer families defined by the 
presence of multiple men with clinically aggressive prostate cancer (having higher grade and/or non-organ-confined disease), additional aggressive prostate cancer loci were identified, including a locus at $22 \mathrm{q} .{ }^{35-41}$ The largest linkage study performed to date to identify susceptibility loci to aggressive prostate cancer was performed by the International Consortium for Prostate Cancer Genetics (ICPCG). ${ }^{41}$ The study focused on 166 (13\%) of 1233 available pedigrees in the ICPCG. The selected families were those that had at least three family members with clinically aggressive disease. Suggestive linkage was found on chromosomes 6p22.3 (LOD 3.0), 11q14.1-14.3 (LOD 2.4) and 20p11.21-q11.21 (LOD 2.5), and a more modest signal was observed at $8 \mathrm{q} 24$. On chromosome 11, the strongest evidence of linkage (LOD 3.31) was observed among pedigrees with an average age at diagnosis of 65 years or younger. In a large follow-up study carried out by the ICPCG ${ }^{42}$ LOD scores over 3.0 were observed at $8 \mathrm{q} 24$ in the vicinity of previously identified common prostate cancer risk variants in families with multiple cases presenting with more aggressive disease. Other than implicating the obvious $8 \mathrm{q} 24$ candidate gene, MYC, the specific genetic variants which may underlie these linkage signals remain undefined, however.

In addition to linkage-based studies, the candidate gene approach has been used to interrogate genes putatively associated with aggressive disease. ${ }^{43-50}$ Lin and colleagues used a candidate approach, genotyping variants in genes associated with various aspects of prostate cancer biology, such as inflammation, steroid-hormone production and metabolism, DNA repair, circadian rhythm and vitamin D activity. ${ }^{51}$ They investigated 937 SNPs in 156 candidate genes, and founds SNPs in five genes (LEPR, RNASEL, IL4, CRY1 and ARVCF) that showed associations with lethal prostate cancers. While these studies have implicated a number of interesting candidate genes, follow-up studies are necessary to confirm these results and to fully understand their contributions to inherited risk for more aggressive prostate cancer.

One important candidate gene that has been associated reproducibly with more aggressive prostate cancer is BRCA2. Although typically associated with increased risk of breast cancer, BRCA2 is a known risk factor for prostate cancer as well, and recent studies have suggested that men carrying mutations in this gene are not only more likely to have a diagnosis of prostate cancer, they are also more likely to high grade, non-organ confined disease. ${ }^{52-55}$

\section{GWAS FOR PROSTATE CANCER AGGRESSIVENESS LOCI}

While most large scale GWAS studies have not addressed tumor aggressiveness as a primary study endpoint, there have been a number of studies which address this question. ${ }^{56-60}$ Penney et al..$^{56}$ performed a GWAS study, searching for loci associated with death from prostate cancer, although the study was limited by the relatively small number of lethal cases available for study. These authors found no variants significantly associated at a genome wide level with prostate cancer mortality, prompting the suggestion that common genetic determinants of lethal prostate cancer, if they exist, are likely to have odds ratios $<2.0$, and that larger studies would be needed to identify genetic influences on susceptibility for this category of disease. Xu et al. ${ }^{59}$ performed a case-case study, comparing men undergoing radical prostatectomy for more or less aggressive disease as assessed by pathological tumor grade and stage. In total, 4829 and 12205 patients with more and less aggressive disease, respectively, were studied. The frequency of the TT genotype of SNP rs4054823 at 17p12 was found to be consistently higher among patients with more aggressive compared with less aggressive disease in each of seven populations studied, with an overall $P$ value of $2.1 \times 10^{-8}$ under a recessive model. The difference in frequency was largest between patients with high-grade, non-organ-confined disease compared with those with low-grade, organ-confined disease. Although these findings needs to be confirmed, this study suggested that inherited variants predisposing to aggressive but not indolent prostate cancer may exist in the genome, and that such variants may have potential as early markers for risk of aggressive prostate cancer.

\section{SUMMARY}

While it has become abundantly clear that genetic factors play an important role in determining overall prostate cancer susceptibility, to what extent aggressiveness of prostate cancer is genetically determined is still an open question. However, if important genetic determinants of prostate cancer aggressiveness truly exist, studies enlisting the appropriate patient populations (e.g., enabling comparisons of large numbers of aggressive cases with large numbers of non-aggressive cases and controls) combined with increasingly more effective techniques for whole exome/genome DNA sequencing will lead to their rapid identification. Such factors could provide major benefits in terms of identifying men at high risk for developing life-threatening disease and targeting them for prevention (e.g., with 5ARIs), early disease screening, and, when appropriate, treatment.

\section{COMPETING FINANCIAL INTERESTS}

The author declares no competing financial interests.

1 Johns LE, Houlston RS. A systematic review and meta-analysis of familial prostate cancer risk. BJU Int 2003; 91: 789-94.

2 Lichtenstein P, Holm NV, Verkasalo PK, Iliadou A, Kaprio J et al. Environmental and heritable factors in the causation of cancer-analyses of cohorts of twins from Sweden, Denmark, and Finland. N Engl J Med 2000; 343: 78-85.

3 Shuen AY, Foulkes WD. Inherited mutations in breast cancer genes-risk and response. J Mammary Gland Biol Neoplasia 2011; 16: 3-15. Review.

4 Langeberg WJ, Isaacs WB, Stanford JL. Genetic etiology of hereditary prostate cancer. Front Biosci 2007; 12: 4101-10.

5 Ewing CM, Ray AM, Lange EM, Zuhlke KA, Robbins CM et al. Germline mutations in HOXB13 and prostate-cancer risk. N Engl J Med 2012; 366: 141-9.

6 Schork NJ, Murray SS, Frazer KA, Topol EJ. Common vs. rare allele hypotheses for complex diseases. Curr Opin Genet Dev 2009; 19: 212-9.

7 http://www.genome.gov/gwastudies/

8 Gudmundsson J, Sulem P, Manolescu A, Amundadottir LT, Gudbjartsson D et al. Genome-wide association study identifies a second prostate cancer susceptibility variant at 8q24. Nat Genet 2007; 39: 631-7.

9 Gudmundsson J, Sulem P, Steinthorsdottir V, Bergthorsson JT, Thorleifsson G et al. Two variants on chromosome 17 confer prostate cancer risk, and the one in TCF2 protects against type 2 diabetes. Nat Genet 2007; 39: 977-83.

10 Thomas G, Jacobs KB, Yeager M, Kraft P, Wacholder S et al. Multiple loci identified in a genome-wide association study of prostate cancer. Nat Genet 2008; 40: 310-5.

11 Gudmundsson J, Sulem P, Rafnar T, Bergthorsson JT, Manolescu A et al. Common sequence variants on $2 \mathrm{p} 15$ and Xp11.22 confer susceptibility to prostate cancer. Nat Genet 2008; 40: 281-3.

12 Eeles RA, Kote-Jarai Z, Giles GG, Olama AA, Guy M et al. Multiple newly identified loci associated with prostate cancer susceptibility. Nat Genet 2008; 40: 316-21.

13 Duggan D, Zheng SL, Knowlton M, Benitez D, Dimitrov L et al. Two genome-wide association studies of aggressive prostate cancer implicate putative prostate tumor suppressor gene DAB2IP. J Natl Cancer Inst 2007; 99: 1836-44.

14 Easton DF, Eeles RA. Genome-wide association studies in cancer. Hum Mol Genet 2008; 17: R109-15.

15 Zheng SL, Sun J, Wiklund F, Smith S, Stattin P, Li G et al. Cumulative association of five genetic variants with prostate cancer. N Engl J Med 2008; 358: 910-9.

16 Kote-Jarai Z, Olama AA, Giles GG, Severi G, Schleutker J et al. Seven prostate cancer susceptibility loci identified by a multi-stage genome-wide association study. Nat Genet 2011; 43: 785-91.

17 Kader AK, Sun J, Isaacs SD, Wiley KE, Yan G et al. Individual and cumulative effect of prostate cancer risk-associated variants on clinicopathologic variables in 5,895 prostate cancer patients. Prostate 2009; 69: 1195-205.

18 Powell IJ, Bock CH, Ruterbusch JJ, Sakr W. Evidence supports a faster growth rate and/or earlier transformation to clinically significant prostate cancer in black than in white American men, and influences racial progression and mortality disparity. $J$ Urol 2010; 183: 1792-6.

19 Thompson IM, Goodman PJ, Tangen CM, Lucia MS, Miller GJ et al. The influence of finasteride on the development of prostate cancer. N Eng/ J Med2003; 349: 215-24. 
Inherited susceptibility for aggressive prostate cancer

WB Isaac

418

20 Siege R, Naishadham D, Jemal A. Cancer statistics, 2012. CA Cancer J Chin 2012; 62: 10-29.

21 Xu J, Sun J, Kader AK, Lindström S, Wiklund F, Usu FC et al. Estimation of absolute risk for prostate cancer using genetic markers and family history. Prostate 2009; 69: 1565-72.

22 Ply M, Wiklund F, Xu J, Isaacs WB, Eklund M, D'Amato M et al. Polygenic risk score improves prostate cancer risk prediction: results from the Stockholm-1 cohort study. Eur Urol 2011; 60: 21-8.

23 Macinnis RJ, Antoniou AC, Eels RA, Sever G, Al Olama AA et al. A risk prediction algorithm based on family history and common genetic variants: application to prostate cancer with potential clinical impact. Genet Epidemiol 2011 ; 35: 549-56.

24 Gilman EP. Complexities of prostate-cancer risk. N Eng J Med 2008; 358: 961-3.

25 Lindström LS, Hall P, Hartman M, Wiklund F, Grönberg H et al. Familial concordance in cancer survival: a Swedish population-based study. Lancet Oncol 2007; 8: 1001-6.

26 Hemminki K. Familial risk and familial survival in prostate cancer. World J Urol; e-pub ahead of print 25 November; do: 10.1007/s00345-011-0801-1.

27 Schaid DJ. The complex genetic epidemiology of prostate cancer. Hum Mol Genet 2004; 1: 13 Spec No. 1: R103-21.

28 Ostrander EA, Kwon EM, Stanford JL. Genetic susceptibility to aggressive prostate cancer. Cancer Epidemiol Biomarkers Prev 2006; 15: 1761-4.

29 Witte JS, Goddard KA, Cont DV, Elston RC, Lin J et al. Genomewide scan for prostate cancer-aggressiveness loci. Am J Hum Genet 2000; 67: 92-9.

30 Goddard KA, Witte JS, Suarez BK, Catalina WJ, Olson JM. Model-free linkage analysis with covariates confirms linkage of prostate cancer to chromosomes 1 and 4. Am J Hum Genet 2001; 68: 1197-206.

31 Neville PJ, Cont DV, Paris PL, Levin H, Catalonia WJ et al. Prostate cancer aggressiveness locus on chromosome 7q32-q33 identified by linkage and allelic imbalance studies. Neoplasia 2002; 4: 424-31.

32 Slayer SL, Schaid DJ, Cunningham JM, McDonnell SK, Marks AF et al. Confirmation of linkage of prostate cancer aggressiveness with chromosome 19q. Am J Hum Genet 2003; 72: 759-62.

33 Pars T, Wörner S, Kurtz F, Haeussler J, Hautmann RE et al. Linkage of aggressive prostate cancer to chromosome 7q31-33 in German prostate cancer families. Eur J Hum Genet 2003; 11: 17-22.

34 Neville PJ, Cont DV, Krumroy LM, Catalonia WJ, Suarez BK et al. Prostate cancer aggressiveness locus on chromosome segment 19q12-q13.1 identified by linkage and allelic imbalance studies. Genes Chromosomes Cancer 2003; 36: 332-9.

35 Stanford JL, McDonnell SK, Friedrichsen DM, Carlson EE, Kolb S et al. Prostate cancer and genetic susceptibility: a genome scan incorporating disease aggressiveness. Prostate 2006; 66: 317-25.

36 Chang BL, Isaacs SD, Wiley KE, Gillanders EM, Zheng SL et al. Genome-wide screen for prostate cancer susceptibility genes in men with clinically significant disease. Prostate 2005; 64: 356-61.

37 Lane EM, Ho LA, Beebe-Dimmer JL, Wang Y, Gillanders EM et al. Genome-wide linkage scan for prostate cancer susceptibility genes in men with aggressive disease: significant evidence for linkage at chromosome 15q12. Hum Genet 2006; 119: 400-7.

38 Slayer SL, Zarfas KE, Brown WM, Lane EM, McDonnell SK et al. Genome-wide linkage scan for prostate cancer aggressiveness loci using families from the University of Michigan Prostate Cancer Genetics Project. Prostate 2006; 66: 173-9.

39 Christensen GB, Camp NJ, Farnham JM, Cannon-Albright LA. Genome-wide linkage analysis for aggressive prostate cancer in Utah high-risk pedigrees. Prostate 2007; 67: 605-13.

40 Schaid DJ, Stanford JL, McDonnell SK, Suuriniemi M, McIntosh L et al. Genome-wide linkage scan of prostate cancer Gleason score and confirmation of chromosome 19q. Hum Genet 2007; 121: 729-35.

41 Li X, Chen I, Plumper SJ, Suarez BK, Casey G et al. Fine-mapping of prostate cancer aggressiveness loci on chromosome 7q22-35. Prostate 2011; 71: 682-9.
42 Schaid DJ, McDonnell SK, Zarfas KE, Cunningham JM, Hebbring S et al. Pooled genome linkage scan of aggressive prostate cancer: results from the International Consortium for Prostate Cancer Genetics. Hum Genet 2006; 120: 471-85.

43 Cicek MS, Cont DV, Curran A, Neville PJ, Paris PL et al. Association of prostate cancer risk and aggressiveness to androgen pathway genes: SRD5A2, CYP17, and the AR. Prostate 2004; 59: 69-76.

44 Casey G, Neville PJ, Li X, Plumber SJ, Cicek MS et al. Podocalyxin variants and risk of prostate cancer and tumor aggressiveness. Hum Mol Genet 2006; 15: 735-41.

45 Hayes VM, Sever G, Southey MC, Padilla EJ, English DR et al. Macrophage inhibitory cytokine-1 H6D polymorphism, prostate cancer risk, and survival. Cancer Epidemiol Biomarkers Prev 2006; 15: 1223-5.

46 Huang SP, Huang CY, Wu WJ, Pu YS, Chen J et al. Association of vitamin D receptor Fol polymorphism with prostate cancer risk, clinicopathological features and recurrence of prostate specific antigen after radical prostatectomy. Int J Cancer 2006; 119: 1902-7.

47 Sever G, Hayes VM, Neufing P, Padilla EJ, Riley WD et al. Variants in the prostatespecific antigen (PSA) gene and prostate cancer risk, survival, and circulating PSA. Cancer Epidemiol Biomarkers Prev 2006; 15: 1142-7.

48 Cussenot O, Azzouzi AR, Nicolaiew N, Fromont G, Margin P et al. Combination of polymorphisms from genes related to estrogen metabolism and risk of prostate cancers: the hidden face of estrogens. J Chin Oncol 2007; 25: 3596-602.

49 Siltanen S, Wahlfors T, Schindler M, Saramäki OR, Mpindi JP et al. Contribution of ARLTS1 Cys 148Arg (T442C) variant with prostate cancer risk and ARLTS1 function in prostate cancer cells. PLOS ONE 2011; 6: e26595.

50 Sun T, Mary LG, Oh WK, Freedman ML, Pomerantz M et al. Inherited variants in the chemokine CCL2 gene and prostate cancer aggressiveness in a Caucasian cohort. Chin Cancer Res 2011; 17: 1546-52.

51 Lin DW, FitzGerald LM, Eu R, Kwon EM, Zheng SL et al. Genetic variants in the LEPR, $C R Y 1, R N A S E L, I L 4$, and ARVCF genes are prognostic markers of prostate cancerspecific mortality. Cancer Epidemiol Biomarkers Prev 2011; 20: 1928-36.

52 Edwards SM, Evans DG, Hope Q, Norman AR, Barbachano Y et al. Prostate cancer in BRCA2 germline mutation carriers is associated with poorer prognosis. $\mathrm{Br} \mathrm{J}$ Cancer 2010; 103: 918-24.

53 Thorn H, Willems AJ, Niedermayr E, Hon IM, Li J et al. Decreased prostate cancerspecific survival of men with BRCA2 mutations from multiple breast cancer families. Cancer Prev Res (Phila) 2011; 4: 1002-10.

54 Kote-Jarai Z, Leongamornlert D, Saunders E, Tymrakiewicz M, Castro E et al. BRCA2 is a moderate penetrance gene contributing to young-onset prostate cancer: implications for genetic testing in prostate cancer patients. Br J Cancer 2011; 105: 1230-4.

55 Gallagher DJ, Gaudet MM, Pal P, Kirchhoff T, Balistreri L et al. Germline BRCA mutations denote a clinicopathologic subset of prostate cancer. Olin Cancer Res 2010; 16: 2115-21.

56 Penney KL, Dyne S, Schumacher FR, Sinnott JA, Mucci LA et al. Genome-wide association study of prostate cancer mortality. Cancer Epidemiol Biomarkers Prev 2010; 19: 2869-76.

57 Nam RK, Chang W, Siminovitch K, Shlien A, Rattan MW et al. New variants at 10q26 and $15 q 21$ are associated with aggressive prostate cancer in a genome-wide association study from a prostate biopsy screening cohort. Cancer Biol The 2011; 12: $997-1004$.

58 FitzGerald LM, Kwon EM, Conomos MP, Kolb S et al. Genome-wide association study identifies a genetic variant associated with risk for more aggressive prostate cancer. Cancer Epidemiol Biomarkers Prev 2011; 20: 1196-203.

$59 \mathrm{Xu}$ J, Zheng SL, Isaac SD, Wiley KE, Wiklund F et al. Inherited genetic variant predisposes to aggressive but not indolent prostate cancer. Proc Natl Acad Sci USA 2010; 107: 2136-40.

60 Jim G, Sun J, Diu W, Chang Z, Chi LW et al. Genome-wide copy-number variation analysis identifies common genetic variants at $20 \mathrm{p} 13$ associated with aggressiveness of prostate cancer. Carcinogenesis 2011; 32: 1057-62.

Asian Journal of Andrology 